\title{
Effect of Pre-sowing Treatment and Sowing Time on Germination and Early Performance of Melia composita
}

\author{
Rafia Jaan and Kamal Kishor Sood* \\ Division of Agroforestry, Sher-e-kashmir University of Agricultural Sciences and Technology- Jammu, \\ Main Campus-Chatha, Jammu-180 009, India \\ *E-mail: kksood_2000_2000@yahoo.com
}

\begin{abstract}
The pre-sowing treatments (soaking drupes in gibberellic acid $50 \mathrm{ppm}$ for 24 hours, soaking drupes in gibberellic acid 100 ppm for 24 hours, soaking drupes in concentrated $\mathrm{H}_{2} \mathrm{SO}_{4}$ for 10 minutes, soaking drupes in concentrated $\mathrm{H}_{2} \mathrm{SO}_{4}$ for 15 minutes, mechanical scarificationcracking of drupes, soaking drupes in cow dung slurry for 30 days and control-no treatment) were tested to enhance the germination of Melia composita at two sowing time $\left(20^{\text {th }}\right.$ April and $04^{\text {th }}$ May). Germination and most of the growth parameters were recorded highest in mechanical scarification. The sowing of $20^{\text {th }}$ April had significantly higher collar diameter, number of leaves, fresh shoot weight, dry shoot weight, length of primary root, number of secondary roots, fresh root weight, dry root weight, total seedling fresh weight, total seedling dry weight and seedling quality index than the sowing date of $04^{\text {th }}$ May. The interaction of mechanical scarification $x$ sowing- $20^{\text {th }}$ April also had significantly higher values for the most studied growth parameters than the remaining interactions. The study implies that mechanical scarification of drupes of Melia composita and sowing in the month of April is appropriate for obtaining good quality planting stock.
\end{abstract}

Keywords: Seed, Germination, Seedling, Growth, Melia composita

Forests and trees play an important role in people's livelihoods and provide products such as food, fodder, fuel, timber and many other non-timber products in addition to indirect services in the form of contribution to maintenance of ecological balance and nutrient cycling. Forests have now gained importance for their role in storing and recycling the earth's carbon through photosynthesis (Schroeder 1994). Forests also play an important role in conservation of biodiversity (FAO 2020) and they have a key role in alleviating poverty and improving food security (FAO 2003). Forests and trees also make an indirect contribution to food security by helping to maintain the environmental conditions suitable for agricultural production (FAO 2020). There are about 1.6 billion people in the world who rely heavily on the forest resource use for their livelihoods. For the world's poor, trees and forests are a vital part of everyday survival and provide 2.4 billion people with fuel to cook (FAO 2020). Deforestation and forest degradation is continuing at a very faster rate, which have adverse affects on the direct and indirect benefits being derived from the forests.

A plantation of trees is one of the solutions to increase the tree cover. At present, forest plantations and agroforestry farms in many countries are dominated by few species like Eucalyptus, Poplar and Casuarina spp. as major sources of raw materials for pulp and paper. However, these species are now being constrained by poor productivity, pests and diseases and are causing ecological degradation. So, there is an urgent need to diversify forest plantations, which is now dominated by few exotics. To address these concerns, there is a need to explore new potential of tree species, which are fast growing and adapted to wide agro-climatic conditions as alternate sources of tree products and raw materials for pulp and paper. Melia composita is one of such species. The species has been reported to have null allelopathic effect on under storey crops (Kumar et al. 2017, Parmar et al 2018), hence could be a promising species for on-farm and off-farm plantations. Wood of Melia composita is an excellent and highly suitable raw material for wood based industries like paper and plywood industries owing to its natural anti-termite property, high pulp recovery and exceptional fibre strength as compared to traditional raw material (Sarvannan et al. 2013). The species performs exceedingly well attaining the harvestable size within 6-8 years and has a ready and assured market due to its multipurpose utilities. Poor germination is a hurdle in afforestation of this species. Therefore, there is a need to enhance its germination capacity and growth by suitably treating its seed (drupes) to obtain quality planting stock for afforestation programmes. Hence, study was conducted to record the performance of pre-sowing treatment and sowing time on germination and early growth of Melia composita.

\section{MATERIAL AND METHODS}

The present study was conducted at the experimental 
farm of the Division of Agroforestry, Sher-e-Kashmir University of Agricultural Sciences and Technology of Jammu (SKUAST-Jammu), India during the period from April 2019 to November 2019. The experimental site is situated in subtropical, Jammu region of Union Territory of Jammu and Kashmir which is located at an altitude $332 \mathrm{~m}$ above mean sea level with $32^{\circ} 40 \square \mathrm{N}$ latitude and $74^{\circ} 58 \square \mathrm{E}$ longitude. Jammu being a subtropical region experiences hot dry summers, humid rainy season and cold winter months. The summer season usually starts from April and lasts up to June. The maximum temperature rises upto $45^{\circ} \mathrm{C}$ during May to June and minimum falls to $1^{\circ} \mathrm{C}$ during winter. The average annual rainfall ranges from $1000-1200 \mathrm{~mm}$ with $75-80 \%$ of which is received during July to September and rest 20-25\% during winter months of December to February.

Seed collection and experimental design: Drupes for carrying out the experiment were collected from the four year old plantation in February, 2019. The drupes were dried in shade for about a month and different pre-treatment were given to the drupes. The sowing of pre-treated drupes was done on $20^{\text {th }}$ April and $04^{\text {th }}$ May (2019). Light irrigation was applied immediately after each sowing followed by weeding and watering as per requirement till the end of the experiment. The experiment was laid out in Factorial CRD (Complete Randomization Design) having two factors i.e. seven pre-sowing treatments (soaking drupes in gibberellic acid 50 ppm for 24 hours, soaking drupes in gibberellic acid 100 ppm for 24 hours, soaking drupes in concentrated $\mathrm{H}_{2} \mathrm{SO}_{4}$ for 10 minutes, soaking drupes in concentrated $\mathrm{H}_{2} \mathrm{SO}_{4}$ for 15 minutes, mechanical scarification-cracking of drupes, soaking drupes in cow dung slurry for 30 days and control-no treatment) and two above mentioned dates. There were 10 polybags (size $16 \mathrm{~cm} \times 24 \mathrm{~cm}$ filled with sieved soil, sand and FYM in ratio of $1: 1: 1)$ per replication per treatment. For recording data, two plants per treatment per replication were chosen using simple random sampling with replacement method. In this way a total of forty two plants were chosen for data recording. The observations on germination, collar diameter $(\mathrm{mm})$, seedling height $(\mathrm{cm})$, number of leaves, length of primary root $(\mathrm{cm})$, number of secondary roots, fresh shoot weight (g), dry shoot weight (g), fresh root weight $(\mathrm{g})$, dry root weight $(\mathrm{g})$, total seedling fresh weight $(\mathrm{g})$, total seedling dry weight $(\mathrm{g})$, root: shoot ratio (dry weight basis), seedling quality index and sturdiness quotient were recorded in the month of November 30, 2019. The final seed germination was recorded 72 days after sowing when no further germination took place. The shoots and roots of sampled plants were dried in hot air oven at $65^{\circ} \mathrm{C}$ for 48 hours to obtain respective dry weights. After drying, the shoot and root weights of each sampled seedling were recorded using digital electronic balance. Sturdiness quotient was calculated by using the formula given by Rollar (1977) and Seedling quality index was estimated by using the formula given by (Dickson et al 1960). The data was analyzed using the technique of analysis of variance (ANOVA) in accordance with procedure outlined by Gomez and Gomez (1984). The effect of different treatments was tested at 0.05 level of significance.

\section{RESULTS AND DISCUSSION}

Germination: The pre-sowing treatments significantly affected drupe germination (Table 1). The maximum germination (46.68\%) per cent was observed in mechanical scarification, which was statistically at par with treatments of soaking drupes in $\mathrm{GA}_{3} 100$ ppm for 24 hours and soaking drupes in $\mathrm{GA}_{3} 50$ ppm for 24 hours but significantly higher than the remaining treatments. The minimum germination $(20 \%)$ was observed in the cowdung treatment and control (Table 1). The effect of date of sowing on germination was also significant (Table 1). The germination obtained in sowing of $04^{\text {th }}$ May $(50.48 \%)$ was significantly higher than that of $20^{\text {th }}$ April (14.76\%). The interaction of treatment $x$ sowing date exhibited non-significant influence on seed germination (Table 1).

Aboveground parameters: All the aboveground parameters (seedling height, collar diameter, number of leaves, fresh and dry weight) of the seedlings were significantly influenced by the pre-sowing treatment of the drupes (Table 2). The maximum seedling height $(163.18 \mathrm{~cm})$, collar diameter (12.96 mm), number of leaves (24.72), fresh shoot weight $(229.66 \mathrm{~g})$ and dry shoot weight $(75.80 \mathrm{~g})$ was observed in mechanical scarification, which were statistically superior to the respective values in the remaining treatments (Table 2) but collar diameter in mechanical scarification was statistically at par with soaking drupes in concentrated $\mathrm{H}_{2} \mathrm{SO}_{4}$ for 15 minutes but significantly superior to the remaining treatments (Table 2). The number of leaves in mechanical scarification was statistically at par with soaking drupes in $\mathrm{GA}_{3} 50$ ppm for 24 hours, soaking drupes in concentrated $\mathrm{H}_{2} \mathrm{SO}_{4}$ for 15 minutes and soaking of drupes in $\mathrm{H}_{2} \mathrm{SO}_{4}$ for 10 minutes (Table 2).

The interaction effect of pre-sowing treatment $x$ sowing date was also significant with to respect to all the studied aboveground parameters (Table 2). The interaction mechanical scarification $\times 20^{\text {th }}$ April resulted in maximum seedling height $(239.43 \mathrm{~cm})$, collar diameter $(20.90 \mathrm{~mm})$, fresh shoot weight $(345.00 \mathrm{~g}$ ) and dry shoot weight (113.86 g) which were statistically superior to all the remaining interactions. The interaction mechanical scarification $\times 20^{\text {th }}$ April also resulted in maximum number of leaves (35.37) but 
this was statistically at par with interaction soaking drupes in $\mathrm{H}_{2} \mathrm{SO}_{4}$ for 15 minutes $\times 20^{\text {th }}$ April but superior to the remaining interactions.

Belowground parameters: The maximum length of primary root $(26.17 \mathrm{~cm})$, number of secondary roots $(20.80)$, fresh root weight $(80.37 \mathrm{~g})$ and dry root weight $(22.70 \mathrm{~g})$ were recorded in mechanical scarification, which was statistically higher than respective values in the remaining treatments (Table 3). The lowest root length $(7.35 \mathrm{~cm})$, number of secondary roots (8.67), fresh root weight (38.77 g) and dry root weight $(10.41 \mathrm{~g})$ was recorded in control. The length of primary root $(19.60 \mathrm{~cm})$, number of secondary roots (13.71), fresh root weight of $(74.86 \mathrm{~g})$ and dry root weight $(17.50 \mathrm{~g})$ was significantly higher in sowing of $20^{\text {th }}$ April than respective values of $04^{\text {th }}$ May (Table 3).

The interaction of pre-sowing treatment $x$ sowing date also significantly affected all the above ground parameters of the seedlings (Table 3). The interaction mechanical scarification $\times 20^{\text {th }}$ April had the maximum number of secondary roots $(26.00)$, fresh root weight $(131.50 \mathrm{~g})$ and root weight $(35.20 \mathrm{~g})$ which were statistically superior to the remaining interactions. The interaction mechanical scarification $\times 20^{\text {th }}$ April also resulted in longest primary root $(30.23 \mathrm{~cm})$ but was statistically at par with mechanical scarification $\times 20^{\text {th }}$ April but superior to remaining interations (Table 3).
The pre-sowing treatment response on seedling parameters was also the same. All the parameters (except root: shoot ratio and sturdiness quotient) were highest in treatment mechanical scarification. Most of these parameters (seedling height, fresh shoot weight, dry shoot weight, length of primary root, number of secondary roots, fresh root weight, dry root weight, total seedling fresh weight and total seedling dry weight) were statistically superior in mechanical scarification in comparison to remaining all the pre-sowing treatments. The germination per cent in mechanical scarification was highest but it was statistically at par with soaking of drupes in $\mathrm{GA}_{3} 100$ ppm for 24 hours and soaking of drupes in $\mathrm{GA}_{3} 50$ ppm for 24 hours. Highest collar diameter was observed in mechanical scarification but it was at par with that of soaking drupes in $\mathrm{H}_{2} \mathrm{SO}_{4}$ for 15 minutes. In treatment mechanical scarification, the number of leaves was highest but at par with soaking of drupes in $\mathrm{GA}_{3} 50$ ppm for 24 hours, soaking drupes in $\mathrm{H}_{2} \mathrm{SO}_{4}$ for 15 minutes and soaking drupes in $\mathrm{H}_{2} \mathrm{SO}_{4}$ for 15 minutes. Similarly seedling quality index in mechanical scarification was observed to be highest but at par with cowdung. Luna et al. (2014) also described mechanical scarification as one of the effective pre-sowing treatments to break physical dormancy caused by hard seed coat.

Mechanical scarification was found to be most effective

Table 1. Effect of pre-sowing treatment and sowing date on germination percentage*

\begin{tabular}{|c|c|c|c|}
\hline \multirow[t]{2}{*}{ Treatment } & \multicolumn{2}{|c|}{ Sowing date } & \multirow[t]{2}{*}{ Mean } \\
\hline & $20^{\text {th }}$ April & $4^{\text {th }}$ May & \\
\hline Soaking drupes in $\mathrm{GA}_{3} 50 \mathrm{ppm}$ for $24 \mathrm{hrs}$ & $\begin{array}{c}10.00 \\
(18.43)\end{array}$ & $\begin{array}{c}63.33 \\
(53.13)\end{array}$ & $\begin{array}{c}36.67 \\
(35.78)\end{array}$ \\
\hline Soaking of drupes in $\mathrm{GA}_{3} 100 \mathrm{ppm}$ for $24 \mathrm{hrs}$ & $\begin{array}{c}26.66 \\
(30.98)\end{array}$ & $\begin{array}{c}66.67 \\
(55.05)\end{array}$ & $\begin{array}{c}46.67 \\
(43.02)\end{array}$ \\
\hline Soaking of drupes in conc. $\mathrm{H}_{2} \mathrm{SO}_{4}$ for 10 minutes & $\begin{array}{c}10.00 \\
(18.43)\end{array}$ & $\begin{array}{c}40.00 \\
(38.83)\end{array}$ & $\begin{array}{r}25.00 \\
(28.63)\end{array}$ \\
\hline Soaking drupes in conc. $\mathrm{H}_{2} \mathrm{SO}_{4}$ for 15 minutes & $\begin{array}{c}16.67 \\
(23.35)\end{array}$ & $\begin{array}{c}43.00 \\
(41.05)\end{array}$ & $\begin{array}{c}30.00 \\
(32.20)\end{array}$ \\
\hline Mechanical scarification & $\begin{array}{c}20.00 \\
(26.06)\end{array}$ & $\begin{array}{c}73.33 \\
(58.98)\end{array}$ & $\begin{array}{c}46.68 \\
(43.52)\end{array}$ \\
\hline Cowdung & $\begin{array}{c}10.00 \\
(18.43)\end{array}$ & $\begin{array}{c}30.00 \\
(33.19)\end{array}$ & $\begin{array}{c}20.00 \\
(25.81)\end{array}$ \\
\hline Control- no treatment & $\begin{array}{c}10.00 \\
(18.43)\end{array}$ & $\begin{array}{c}36.67 \\
(37.13)\end{array}$ & $\begin{array}{c}23.33 \\
(27.77)\end{array}$ \\
\hline Mean & $\begin{array}{c}14.76 \\
(22.02)\end{array}$ & $\begin{array}{c}50.48 \\
(45.34)\end{array}$ & \\
\hline Effect & & $C D(p=0.05)$ & $\pm \mathrm{SE}(\mathrm{m})$ \\
\hline Treatment & & $\begin{array}{l}12.77 \\
(7.99)\end{array}$ & $\begin{array}{c}4.41 \\
(2.76)\end{array}$ \\
\hline Sowing date & & $\begin{array}{c}6.83 \\
(4.27)\end{array}$ & $\begin{array}{c}2.36 \\
(1.47)\end{array}$ \\
\hline Treatment $\mathrm{x}$ Sowing date & & $\begin{array}{c}\text { N.S } \\
-\end{array}$ & $\begin{array}{c}6.24 \\
(3.90) \\
\end{array}$ \\
\hline
\end{tabular}

${ }^{*}$ Figures in parenthesis are transformed (angular) values 
Table 2. Effect of pre-sowing treatment and sowing date on aboveground parameters

\begin{tabular}{|c|c|c|c|c|}
\hline \multirow[t]{2}{*}{ Treatment } & \multirow[t]{2}{*}{ Parameter } & \multicolumn{2}{|c|}{ Sowing date } & \multirow[t]{2}{*}{ Mean } \\
\hline & & $20^{\text {th }}$ April & $4^{\text {th }}$ May & \\
\hline Soaking drupes in $\mathrm{GA}_{3} 50 \mathrm{ppm}$ for $24 \mathrm{hrs}$ & $\begin{array}{c}\text { SH } \\
\text { CD } \\
\text { NL } \\
\text { FSW } \\
\text { DSW }\end{array}$ & $\begin{array}{c}43.33 \\
8.02 \\
28.33 \\
128.43 \\
37.50\end{array}$ & $\begin{array}{c}138.17 \\
7.37 \\
20.83 \\
150.77 \\
39.20\end{array}$ & $\begin{array}{c}90.75 \\
7.69 \\
24.58 \\
139.60 \\
38.35\end{array}$ \\
\hline Soaking of drupes in $\mathrm{GA}_{3} 100 \mathrm{ppm}$ for $24 \mathrm{hrs}$ & $\begin{array}{c}\text { SH } \\
\text { CD } \\
\text { NL } \\
\text { FSW } \\
\text { DSW }\end{array}$ & $\begin{array}{c}85.57 \\
8.63 \\
20.20 \\
144.13 \\
34.16\end{array}$ & $\begin{array}{c}121.27 \\
6.90 \\
19.93 \\
151.38 \\
37.85\end{array}$ & $\begin{array}{c}103.42 \\
7.77 \\
20.07 \\
147.75 \\
36.00\end{array}$ \\
\hline Soaking of drupes in conc. $\mathrm{H}_{2} \mathrm{SO}_{4}$ for 10 minutes & $\begin{array}{c}\text { SH } \\
\text { CD } \\
\text { NL } \\
\text { FSW } \\
\text { DSW }\end{array}$ & $\begin{array}{c}128.00 \\
8.47 \\
24.33 \\
135.89 \\
44.80\end{array}$ & $\begin{array}{c}122.40 \\
6.38 \\
20.50 \\
145.53 \\
40.75\end{array}$ & $\begin{array}{c}125.20 \\
7.42 \\
22.42 \\
140.71 \\
42.76\end{array}$ \\
\hline Soaking drupes in conc. $\mathrm{H}_{2} \mathrm{SO}_{4}$ for 15 minutes & $\begin{array}{c}\text { SH } \\
\text { CD } \\
\text { NL } \\
\text { FSW } \\
\text { DSW }\end{array}$ & $\begin{array}{c}164.53 \\
16.33 \\
33.10 \\
250.01 \\
58.50\end{array}$ & $\begin{array}{c}100.40 \\
6.36 \\
15.80 \\
145.07 \\
33.37\end{array}$ & $\begin{array}{c}132.47 \\
11.34 \\
24.45 \\
197.54 \\
45.94\end{array}$ \\
\hline Mechanical scarification & $\begin{array}{c}\text { SH } \\
\text { CD } \\
\text { NL } \\
\text { FSW } \\
\text { DSW }\end{array}$ & $\begin{array}{c}239.43 \\
20.90 \\
35.37 \\
345.00 \\
113.86\end{array}$ & $\begin{array}{c}86.93 \\
5.01 \\
14.07 \\
114.33 \\
37.73\end{array}$ & $\begin{array}{c}163.18 \\
12.96 \\
24.72 \\
229.66 \\
75.80\end{array}$ \\
\hline Cowdung & $\begin{array}{c}\text { SH } \\
\text { CD } \\
\text { NL } \\
\text { FSW } \\
\text { DSW }\end{array}$ & $\begin{array}{c}23.17 \\
9.83 \\
27.67 \\
168.17 \\
42.20\end{array}$ & $\begin{array}{c}54.67 \\
4.60 \\
13.07 \\
110.92 \\
26.64\end{array}$ & $\begin{array}{c}38.92 \\
7.22 \\
20.37 \\
139.55 \\
34.42\end{array}$ \\
\hline Control- no treatment & $\begin{array}{c}\text { SH } \\
\text { CD } \\
\text { NL } \\
\text { FSW } \\
\text { DSW }\end{array}$ & $\begin{array}{c}24.33 \\
7.17 \\
17.67 \\
167.26 \\
43.49\end{array}$ & $\begin{array}{c}85.57 \\
3.79 \\
13.10 \\
88.45 \\
20.35\end{array}$ & $\begin{array}{c}54.95 \\
5.47 \\
15.38 \\
127.85 \\
31.92\end{array}$ \\
\hline Mean & $\begin{array}{c}\text { SH } \\
\text { CD } \\
\text { NL } \\
\text { FSW } \\
\text { DSW }\end{array}$ & $\begin{array}{c}101.21 \\
11.24 \\
26.67 \\
191.27 \\
53.50\end{array}$ & $\begin{array}{c}101.34 \\
5.87 \\
16.76 \\
129.49 \\
33.70\end{array}$ & \\
\hline Effect & & & $C D(p=0.05)$ & $\pm \mathrm{SE}(\mathrm{m})$ \\
\hline Treatments & & & $\begin{array}{c}4.50 \\
2.42 \\
3.08 \\
16.69 \\
1.27\end{array}$ & $\begin{array}{l}15.55 \\
0.84 \\
1.06 \\
5.76 \\
0.23\end{array}$ \\
\hline Sowing date & & & $\begin{array}{l}\text { N.S } \\
1.30 \\
1.64 \\
8.92 \\
0.67\end{array}$ & $\begin{array}{l}8.31 \\
0.44 \\
0.57 \\
3.07 \\
0.43\end{array}$ \\
\hline Treatments $\times$ Sowing date & & & $\begin{array}{c}63.75 \\
3.42 \\
4.36 \\
23.60 \\
1.79\end{array}$ & $\begin{array}{c}22.00 \\
1.18 \\
1.50 \\
8.14 \\
0.62\end{array}$ \\
\hline
\end{tabular}

SH-Seedling height (cm), CD-Collar diameter (mm), NL- Number of leaves, FSW-Fresh shoot weight (g), DSW-Dry shoot weight (g) 
Table 3. Effect of pre-sowing treatment and sowing date on below ground parameters

\begin{tabular}{|c|c|c|c|c|}
\hline \multirow[t]{2}{*}{ Treatment } & \multirow[t]{2}{*}{ Parameter } & \multicolumn{2}{|c|}{ Sowing date } & \multirow[t]{2}{*}{ Mean } \\
\hline & & $20^{\text {th }}$ April & $4^{\text {th }}$ May & \\
\hline \multirow{4}{*}{$\begin{array}{l}\text { Soaking drupes in } \mathrm{GA}_{3} 50 \text { ppm for } 24 \\
\text { hrs }\end{array}$} & LPR & 20.20 & 11.40 & 15.80 \\
\hline & NSR & 6.66 & 16.67 & 11.67 \\
\hline & FRW & 55.65 & 45.80 & 50.75 \\
\hline & DRW & 11.65 & 11.45 & 11.55 \\
\hline \multirow{4}{*}{$\begin{array}{l}\text { Soaking of drupes in } \mathrm{GA}_{3} 100 \mathrm{ppm} \text { for } \\
24 \mathrm{hrs}\end{array}$} & LPR & 26.13 & 22.13 & 24.13 \\
\hline & NSR & 16.66 & 13.67 & 15.67 \\
\hline & FRW & 59.45 & 42.32 & 50.88 \\
\hline & DRW & 15.82 & 11.43 & 13.63 \\
\hline \multirow{4}{*}{$\begin{array}{l}\text { Soaking of drupes in conc. } \mathrm{H}_{2} \mathrm{SO}_{4} \text { for } \\
10 \text { minutes }\end{array}$} & LPR & 10.43 & 12.73 & 11.58 \\
\hline & NSR & 13.00 & 10.00 & 11.50 \\
\hline & FRW & 55.38 & 40.19 & 47.79 \\
\hline & DRW & 11.60 & 14.10 & 12.85 \\
\hline \multirow{4}{*}{$\begin{array}{l}\text { Soaking drupes in } \mathrm{H}_{2} \mathrm{SO}_{4} \text { for } 15 \\
\text { minutes }\end{array}$} & LPR & 30.23 & 11.20 & 20.72 \\
\hline & NSR & 13.66 & 5.67 & 9.67 \\
\hline & FRW & 100.30 & 37.18 & 68.74 \\
\hline & DRW & 20.00 & 16.26 & 18.13 \\
\hline \multirow[t]{4}{*}{ Mechanical scarification } & LPR & 30.20 & 22.13 & 26.17 \\
\hline & NSR & 26.00 & 15.60 & 20.80 \\
\hline & FRW & 131.50 & 29.23 & 80.37 \\
\hline & DRW & 35.20 & 10.18 & 22.70 \\
\hline \multirow[t]{4}{*}{ Cowdung } & LPR & 10.00 & 10.00 & 10.00 \\
\hline & NSR & 18.00 & 4.00 & 11.00 \\
\hline & FRW & 69.30 & 28.76 & 49.30 \\
\hline & DRW & 16.68 & 10.35 & 15.52 \\
\hline \multirow[t]{4}{*}{ Control- no treatment } & LPR & 10.00 & 4.70 & 7.35 \\
\hline & NSR & 10.00 & 7.33 & 8.67 \\
\hline & FRW & 52.45 & 25.09 & 38.77 \\
\hline & DRW & 11.54 & 9.28 & 10.41 \\
\hline \multirow[t]{4}{*}{ Mean } & LPR & 19.60 & 13.47 & \\
\hline & NSR & 13.71 & 9.95 & \\
\hline & FRW & 74.86 & 35.51 & \\
\hline & DRW & 17.50 & 11.86 & \\
\hline Effect & & & $\mathrm{CD}_{0.05}$ & $\square \mathrm{SE}(\mathrm{m})$ \\
\hline \multirow[t]{4}{*}{ Treatments } & & LPR & 0.23 & 0.79 \\
\hline & & NSR & 1.41 & 0.48 \\
\hline & & FRW & 2.05 & 0.70 \\
\hline & & DRW & 1.54 & 0.53 \\
\hline \multirow[t]{4}{*}{ Sowing date } & & LPR & 0.12 & 0.04 \\
\hline & & NSR & 0.75 & 0.26 \\
\hline & & FRW & 1.11 & 0.37 \\
\hline & & DRW & 0.82 & 0.28 \\
\hline \multirow[t]{4}{*}{ Treatment $x$ Sowing date } & & LPR & 0.33 & 0.11 \\
\hline & & NSR & 2.00 & 0.69 \\
\hline & & FRW & 2.90 & 1.00 \\
\hline & & DRW & 2.17 & 0.74 \\
\hline
\end{tabular}

LPR- Length of primary root (cm), NSR-Number of secondary roots, FRW-Fresh root weight (g), DRW-Dry root weight (g) 
Table 4. Effect of pre-sowing treatment and sowing date on whole seedling parameters

\begin{tabular}{|c|c|c|c|c|}
\hline \multirow[t]{2}{*}{ Treatment } & \multirow[t]{2}{*}{ Parameter } & \multicolumn{2}{|c|}{ Sowing date } & \multirow[t]{2}{*}{ Mean } \\
\hline & & $20^{\text {th }}$ April & $4^{\text {th }}$ May & \\
\hline \multirow{5}{*}{$\begin{array}{l}\text { Soaking drupes in } \mathrm{GA}_{3} 50 \mathrm{ppm} \text { for } 24 \\
\text { hrs }\end{array}$} & TSFW & 184.08 & 196.57 & 190.33 \\
\hline & TSDW & 49.15 & 50.65 & 49.90 \\
\hline & $\mathrm{R}: \mathrm{S}$ ratio & 0.31 & 0.29 & 0.30 \\
\hline & $S Q$ & 5.90 & 17.60 & 11.75 \\
\hline & SQI & 8.62 & 2.28 & 5.58 \\
\hline \multirow{5}{*}{$\begin{array}{l}\text { Soaking of drupes in } \mathrm{GA}_{3} 100 \mathrm{ppm} \text { for } \\
24 \mathrm{hrs}\end{array}$} & TSFW & 204.18 & 193.70 & 198.94 \\
\hline & TSDW & 49.98 & 49.28 & 49.63 \\
\hline & $\mathrm{R}: \mathrm{S}$ ratio & 0.46 & 0.29 & 0.37 \\
\hline & $S Q$ & 9.93 & 17.90 & 13.91 \\
\hline & SQI & 4.41 & 2.36 & 3.25 \\
\hline \multirow{5}{*}{$\begin{array}{l}\text { Soaking of drupes in conc. } \mathrm{H}_{2} \mathrm{SO}_{4} \text { for } 10 \\
\text { minutes }\end{array}$} & TSFW & 191.27 & 185.72 & 188.50 \\
\hline & TSDW & 56.40 & 54.85 & 55.63 \\
\hline & $\mathrm{R}: \mathrm{S}$ ratio & 0.26 & 0.35 & 0.31 \\
\hline & $S Q$ & 16.39 & 19.51 & 17.95 \\
\hline & SQI & 2.97 & 0.49 & 1.73 \\
\hline \multirow[t]{5}{*}{ Soaking drupes in $\mathrm{H}_{2} \mathrm{SO}_{4}$ for 15 minutes } & TSFW & 125.31 & 182.25 & 153.78 \\
\hline & TSDW & 78.50 & 49.63 & 64.07 \\
\hline & $\mathrm{R}: \mathrm{S}$ ratio & 0.30 & 0.45 & 0.37 \\
\hline & $\mathrm{SQ}$ & 10.03 & 16.35 & 13.19 \\
\hline & SQI & 6.03 & 2.78 & 4.40 \\
\hline \multirow[t]{5}{*}{ Mechanical scarification } & TSFW & 476.50 & 143.56 & 310.03 \\
\hline & TSDW & 149.06 & 47.91 & 98.49 \\
\hline & $\mathrm{R}: \mathrm{S}$ ratio & 0.32 & 0.33 & 0.33 \\
\hline & $\mathrm{SQ}$ & 11.82 & 17.51 & 14.66 \\
\hline & SQI & 12.07 & 2.56 & 7.32 \\
\hline \multirow[t]{5}{*}{ Cowdung } & TSFW & 237.47 & 139.68 & 188.56 \\
\hline & TSDW & 58.88 & 36.99 & 47.94 \\
\hline & $\mathrm{R}: \mathrm{S}$ ratio & 0.36 & 0.38 & 0.37 \\
\hline & $\mathrm{SQ}$ & 2.37 & 11.98 & 7.18 \\
\hline & SQI & 10.15 & 2.27 & 6.21 \\
\hline \multirow[t]{5}{*}{ Control-no treatment } & TSFW & 219.71 & 113.54 & 166.63 \\
\hline & TSDW & 60.81 & 29.60 & 45.21 \\
\hline & $\mathrm{R}: \mathrm{S}$ ratio & 0.27 & 0.45 & 0.36 \\
\hline & $S Q$ & 3.39 & 23.20 & 13.29 \\
\hline & SQI & 0.95 & 1.19 & 1.07 \\
\hline \multirow[t]{5}{*}{ Mean } & TSFW & 234.07 & 165.00 & \\
\hline & TSDW & 71.83 & 45.56 & \\
\hline & $\mathrm{R}: \mathrm{S}$ ratio & 0.33 & 0.36 & \\
\hline & $\mathrm{SQ}$ & 8.55 & 17.72 & \\
\hline & SQI & 6.46 & 1.20 & \\
\hline Effect & & & $C D(p=0.05)$ & $\pm \mathrm{SE}(\mathrm{m})$ \\
\hline
\end{tabular}


Table 4. Effect of pre-sowing treatment and sowing date on whole seedling parameters

\begin{tabular}{|c|c|c|c|c|}
\hline \multirow[t]{2}{*}{ Treatment } & \multirow[t]{2}{*}{ Parameter } & \multicolumn{2}{|c|}{ Sowing date } & \multirow[t]{2}{*}{ Mean } \\
\hline & & $20^{\text {th }}$ April & $4^{\text {th }}$ May & \\
\hline \multirow[t]{5}{*}{ Treatments } & TSFW & & 20.46 & 7.05 \\
\hline & TSDW & & 1.87 & 0.64 \\
\hline & $\mathrm{R}: \mathrm{S}$ ratio & & 0.04 & 0.01 \\
\hline & $\mathrm{SQ}$ & & 5.08 & 1.75 \\
\hline & SQI & & 1.60 & 0.55 \\
\hline \multirow[t]{5}{*}{ Sowing date } & TSFW & & 9.98 & 3.80 \\
\hline & TSDW & & 1.00 & 0.34 \\
\hline & $\mathrm{R}: \mathrm{S}$ ratio & & 0.02 & 0.01 \\
\hline & $S Q$ & & 2.72 & 0.94 \\
\hline & SQI & & 0.85 & 0.29 \\
\hline \multirow[t]{5}{*}{ Treatment $x$ Sowing date } & TSFW & & 29.66 & 10.23 \\
\hline & TSDW & & 2.65 & 0.91 \\
\hline & $\mathrm{R}: \mathrm{S}$ ratio & & 0.06 & 0.02 \\
\hline & $S Q$ & & N.S & 2.48 \\
\hline & SQI & & 2.27 & 0.78 \\
\hline
\end{tabular}

TSFW-Total seedling fresh weight (g), TSDW-Total seedling dry weight (g), R:S- Root: shoot ratio, SQ-Sturdiness quotient, SQI- Seedling quality index

in enhancing fresh shoot weight, dry shoot weight, length of primary root, number of secondary roots, fresh root weight, dry root weight, total seedling fresh weight and total seedling dry weight. The maximum biomass parameters in mechanically scarified drupes in the current study might be due to early initiation of germination on account of faster absorption of water and gaseous exchange and consequently longer period of time for growth compared to remaining treatments. According to Jaenicke (1999) a small sturdiness quotient indicates a sturdy plant with higher expected chances of survival especially on windy or dry sites. Usually sturdiness quotient of less than six is desirable. In current study none of treatments had sturdiness quotient less than 6 . The maximum root: shoot $(0.37)$ was recorded in soaking of drupes in $\mathrm{GA}_{3} 100$ ppm for 24 hours and was at par with soaking drupes in $\mathrm{H}_{2} \mathrm{SO}_{4}$ for 15 minutes, cowdung and mechanical scarification. Root:shoot ratio reflects the capacity of the roots to support the above ground biomass not only for anchorage but also in absorbing nutrients and water from the soil. A high root: shoot ratio indicates high absorption and storage capacity of water, which is an advantage, especially in conditions of limited soil moisture (Takoutsing et al 2016). In current study, all these treatments i.e. soaking of drupes in $\mathrm{GA}_{3} 100 \mathrm{ppm}$ for 24 hours and soaking drupes in $\mathrm{H}_{2} \mathrm{SO}_{4}$ for 15 minutes, cowdung and mechanical scarification were equally good with respect to root:shoot ratio. The seedling quality index was highest in mechanical scarification in current study. Seedling quality index is overall indicator of seedling performance (Annapurna et al 2004). Since the majority of growth parameters were maximum in mechanical scarification thereby, the seedling quality index was also highest in this treatment. This implies that overall the mechanical scarification is overall superior treatment.

\section{CONCLUSION}

The mechanical scarification of drupes of Melia composita needs to be carried out before sowing to enhance it's germination and growth. Sowing of the seeds in the month of April is appropriate than further delay.

\section{REFERENCES}

Annapurna D, Rathore TS and Joshi G 2004. Effect of container type and size on the growth and quality of seedlings of Indian sandalwood (Santalum album L.). Australian Forestry 67(2): 82-87.

Dickson A, Leaf AL and Hosner JF 1960. Quality appraisal of white Supruce and white Pine seedling stock in nurseries. Forestry Chronicle 36(4): 10-13.

FAO 2003. State of World's Forest 2003. FAO, Rome.

FAO 2020. The State of World's Forests. Forests, Biodiversity and People. FAO, Rome, Italy.

Gomez KA and Gomez AA 1984. Statistical Procedures for Agricultural Research. John Wlley and Sons, Newyork, U.S.A.

Jaenicke H 1999. Good Tree Nursery Practices. Practical Guidelines for Community Nurseries. International Centre for Research in Agroforestry, Nairobi, Kenya.

Kumar D, Thakur NS and Gunaga RP 2017. Transient allelopathic propensity of Melia composita Willd. Leaf litter on chickpea (Cicer arietinum L). Indian Journal of Ecology 44: 443-450. 
Luna T, Wilkinson KM And Dumroese RK 2014. Seed germination and sowing options, pp.163-183. In: KM Wilkinson, TD Landis, DL Haase, BF Daley, RK Dumroese (eds), Tropical Nursery Manual: A Guide to Starting and Operating A Nursery For Native and Traditional Plants. Agriculture Handbook 732. Washington, DC: US Department of Agriculture, Forest Service.

Mohammad N, Rajkuamr M, Singh K, Nain NPS, Singh S, Rao GR and Prakasham U 2021. Spacing, pit size and irrigation influence early growth performances of forest tree species. Journal of Tropical Forest Science 33(1): 69-76.

Parmar AG, Thakur NS and Gunaga RP 2018. Melia dubia Cav. leaf litter allelochemicals have ephemeral allelopathic proclivity. Agroforestry Systems 93(4): 1347-1360.

Rollar KJ 1977. Suggested Minimum Standards for Containerised

Received 22 September, 2021; Accepted 10 January, 2022
Seedlings in Nova Scotia. Report M-X. Canadian Forestry Service Department of Environment Information. Frederiction, New Brunswisk, Canada.

Sarvannan V, Parthiban, KT, Kumar, P and Marimuthu, P 2013. Wood characterization studies on Melia dubia Cav. for pulp and paper industry at different age gradation. Research Journal of Recent Sciences 2(ISC2012): 183-188.

Schroeder P 1994. Carbon storage benefits of agroforestry. Agroforestry Systems 27: 89-97.

Takoutsing B, Weber J, Aynekulu E, Martín JAR, Shepherd K, Sila A and Diby L 2016. Assessment of soil health indicators for sustainable production of maize in smallholder farming systems in the highlands of Cameroon. Geoderma 276: 6473. 